\title{
Using Deep Learning for Arabic Writer Identification
}

\author{
Shaza Maaz \\ Aleepo, Syria \\ Computer Engineering Department, College of \\ Electrical and Electronic Engineering, University of \\ Aleppo
}

\author{
Hazem Issa \\ Aleepo, Syria \\ Computer Engineering Department, College of \\ Electrical and Electronic Engineering, University of \\ Aleppo
}

\begin{abstract}
Identification of persons is mainly through the physiological characteristics like fingerprints, face, iris, retina, and hand geometry and the behavioral characteristics like a voice, signature, and handwriting. Identifying the author of a handwritten document has been an active field of research over the past few years and it used in many applications as in biometrics, forensics and historical document analysis. This research presents the study and implementation of the stages of writer identification, starting from data acquisition, and then augmente the data through programming an algorithm that generate a large number of texts from the set of texts available within the database, finally building a convolutional Neural Network (CNN)) Which is useful for extracting features information and then classification the data, therefore, the features are not needed to pre-define. The experiments in this study were conducted on images of Arabic handwritten documents from ICFHR2012 dataset of 202 writer, and each writer have 3 text. The proposed method achieved a classification accuracy of $98.2426 \%$.
\end{abstract}

\section{General Terms}

Pattern Recognition, Writer identification

\section{Keywords}

Arabic handwriting, data augmentation, writer identification, deep learning, convolutional Neural Network

\section{INTRODUCTION}

The process of Identifying people includes the process of identifying the characteristics of each person such as fingerprints, face, iris, and handwriting because each person has his own unique handwriting style that can be used to identify document writer. Identifying people involve an fields of Artificial intelligence (AI), image processing and pattern recognition, and it contributes in many applications: biometrics, forensics, security, and legal matters, and financial field ... etc[1]. Writer identification was classified into two categories, online and off-line. In on-line case, the features gathered directly from signals that dispatched from digital devices. Further, in off-line case the features gathered from the handwritten text, which it acquired from scanned image. It is considered as more complex than on-line case due to many dynamic features of handwriting are missing, for instance pen-pressure, order of strokes, and writing quickness [2]. The task of classification in pattern recognition is to identify the pattern to a class of the known group of classes, in which features will be extracted from images of scanned texts handwritten and trained it by using a classification algorithm. This study proposed a system for identifying people through Arabic handwritten text by deep learning, which required data augmentation. Beacause of limited number of handwritten text samples, it is almost impossible and unrealistic to collect a large number of samples from different writers. The text is divided into parts and then Gathering all text structures randomly to obtain several lines, which enables us to generate many texts from them, and then design a convolutional neural network, which proves its ability to achieve high accuracy in writer identification. This research includes a number of previous studies on handwriting features extraction, person identification techniques, and some data augmentation techniques.

\section{RELATED WORKS}

In this paragraph, the studies will be discussed that have been done on writer identification and the techniques used so far, with an overview of writer identification systems in several languages, such as Chinese, English, Arabic ... etc.

Researcher Tomai et al presented in 2004 a study using several methods as Character level features and Word level features for extracting features extraction, and Euclidean distances as a classification methodology on texts written in English, the final performance was close to 99\% [3]. In 2009, researcher Zhu et al presented a study in this field using the Shape Codebook and Multi Class SVM Classifier, and their proposed method was evaluated by collecting images of texts written in eight languages (Arabic, Chinese, English, Hindi, Japanese, Korean, Russian and Thai). The Chinese identification rate was much lower $(55.1 \%)$ compared to the eight other languages [4]. Researcher Bangy Li et al in 2009 proposed a method based on combining the static feature and dynamic feature, and then the Nearest Neighbor Classifier was used as a classification methodology. The researchers conducted their study on images of English and Chinese handwriting, the results showed that their proposed method achieves a high degree of accuracy and speed, the writer identification rate was $91.5 \%$ in Chinese texts and $93.6 \%$ in the English texts [5]. In 2013, researcher Djeddi et al proposed a method for writer identifition by relying on a set of feature extracted based on a set of run-length features, which are compared with the well-known state-of-the-art features. Classification is carried out using K-Nearest Neighbors (K-NN) and Support Vector Machines (SVM). The experimental results obtained on a database of 126 writers with 4 samples per writer show that the proposed scheme achieves interesting performances on writer identification and verification in a multi-script environment [6]. A study presented by the researcher Alaei et al in 2014 proposing a method for identifying the writer based on Histogram, using two different sets of handwritten data written in Canadian and English languages.thier experiments were conducted on 228 Canadian-language texts for 57 writers with $92.79 \%$ accuracy for F-measure and 330 texts in English for 55 writers with $26.67 \%$ accuracy for F-measure [7]. In 2015, researcher Thendral et al proposed a study that predicts a person using images of Tamil handwriting, using a new discriminative model with pooled features of handwriting is proposed and implemented using support vector machine. It has been 
reported on $100 \%$ of prediction accuracy by $\mathrm{RBF}$ and polynomial kernel based classification model [8].

The Arabic writer identification was not covered as widely as English or Chinese writer identification until the last few years, and the first study goes back to the proposal of the researcher AL-Zoubeidy et al in 2005 using multi -channel Gabor filtering and gray scale co-occurrence matrices to distinguish writer from his writing style [9]. In 2007, Gazzah et al combined local features with global features, and used the Modular Multilayer Perceptron (MLP) classifier. Experiments have shown that the accuracy of identifying the writer reaches the best levels of performance at a rate of $95.68 \%$ [10]. Chen et al in 2010, they proposed a method for detecting and removing ruling lines from handwritten documents and testing that it is usefull to identify Arabic writer through a series of experiments. Initial results indicate that ruling lines have different properties, for example when collecting thickness and spacing. Etc., removing them significantly improves identification performance, this study use global features and Support Vector Machines are used as classification methodology [11]. Fouad and Volke in 2014 proposed a system for Arabic writer identifition using only 21 feature, and Gaussian Mixture Models (GMMs) were also used as the kernel of this system, where GMMs provide a strong representation of the distribution of the extracted features using a fixed-length sliding window It is constructed and trained using images of words and texts for writer [12]. In 2012, Meslatiet et al presented a study for arabic writer identificatio by using the edge hinge distribution and runlengths distribution to extract features. To define the writer, a nearest-neighbor classification was used. It was found that using the run-lengths distribution method give good discriminative information [13]. Djeddi et al in 2014 conducted their study on handwritten texts of 1,000 writers using three methods to extract features: run-length distribution, edge-hinge distribution, and edge-direction features. They used Multiclass SVM (Support Vector Machine), and then had an identification rate of $84.10 \%$ [14]. Researcher Bennour presented in 2018 a study on texts written by 275 writers and each writer had five texts using Gray Level Co-occurrence Matrices to extract the characteristics and Clonal Selection Classification Algorithm (CSCA) in the classification phase. The classification accuracy achieved by this study was $95.38 \%$ [15]. Dengel et al presented the Arabic text recognition system using the (KHATT) database. This study contributed mainly in three aspects i.e., (1) pre-processing, (2) deep learning based approach, and (3) data-augmentation by creating copies of the data. The first instance is the blur version of the original instance. The second instance emphasizes on the contours of the text-line. The third and fourth instances represent enhanced edges and bleed through effect. Finally, the fifth instance represent distorted image having $25 \%$ pixels as off. This study has achieved an accuracy of $80.02 \%$ [16]. Rehman et al applied techniques for data augmentation to improve performance like contours, negatives, and sharpness using textline images of target data set. The sliding window approach was used to make patches as an input unit to the CNN model. The AlexNet architecture was employed to extract discriminating visual features from multiple representations of image patches generated by enhanced preprocessing techniques. The extracted features from patches are then fed to a support vector machine classifier. they realized the highest accuracy to $92.78 \%$ on English, $92.20 \%$ on Arabic, and $88.11 \%$ on the combination of Arabic and English, respectively [17].

\section{PROPOSED SYSTEM}

In this paragraph, the Arabic writer identification is presented. First, Image Acquisition of handwritten texts in Arabic, Secondly, the data-augmentation technique for increasing the input data images, finally, training process and the experimental are presented.

\subsection{Image Acquisition}

The study was conducted on an ICFHR2012 database downloaded from Kaggle for 202 writers, each writer had three different handwritten texts in Arabic, and the images were at a resolution of 600 DPP binary [18].

\subsection{Pre-processing}

The database did not need to be pre-processed so it was binary images with white background and text in black, this data just need to be increased.

\subsection{Data Augmentation}

The handwritten documents may be limited, which prompted a number of researchers to techniques for increasing the data. Training the neural network need large number of samples, so an algorithm was programed to increase the number of images of handwritten texts in the Arabic language, especially the data in this study contains only three texts for each writer, and this number is not sufficient to train the proposed neural network. In addition, the fact that the images have different large sizes and this does not suit to the proposed network and also to avoid the problem of out of memory.

After reviewing some research in this field, a method has been programmed to increase the data in accordance with the data, which are used, and through the following stages:

1) Divide the text into words or combination of word. After reviewing the two studies presented by Tang et al, and Doermann et al presenting a method for dividing the text into words according to the following stages, which are shown in "Figure 1":

a) Converting the orginal image into a binary image by using Otsu's algorithm [See "Figure.1(b)"].

b) Filtering a binary image with an isotropic LoG filter to get the filtered image [See "Figure.1(c)"].

c) Binarizing filtered image to get a binary image by using the threshold obtained by Otsu's algorithm [See " Figure. 1(d)"].

d) Assigning each connected-component in binary image to the nearest connected region of image from stage (c) to form semi-word regions (SWRs), which are labeled with different colors in " Figure. $1(\mathrm{e}) "$.

e) Merging the SWRs to get the word regions (WRs) according to the distances between the adjacent SWRs [See " Figure. 1(f)"].

f) Splitting the overlapping connected-components (OCCs) which run along multiple text lines from the middle line of these OCCs' boundary box [See " Figure. 1(g)"] [19,20]. 


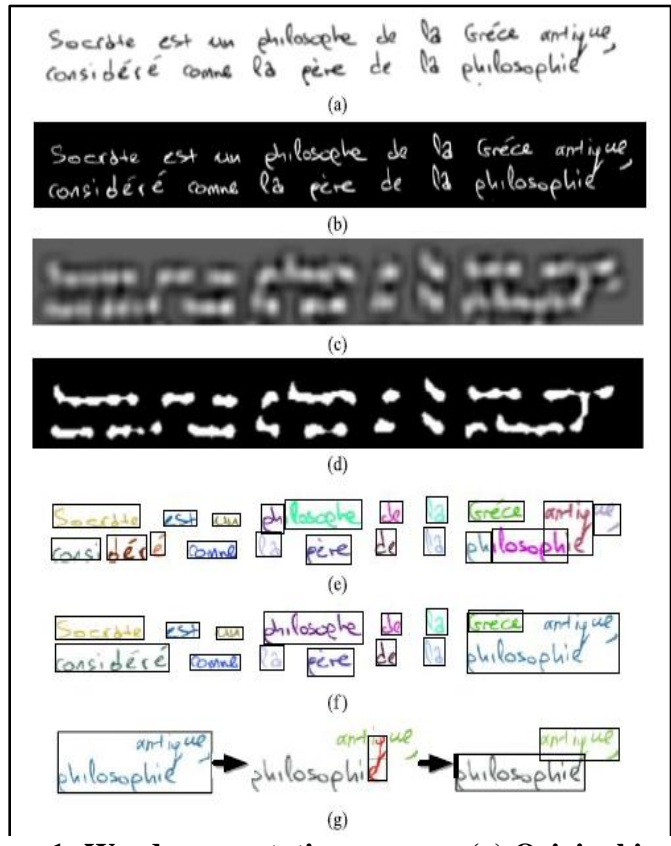

Figure 1: Word segmentation process. (a) Original image

(I). (b) Binary image. (c) Filtered image. (d) Binary

Filtered image. (e) Semi-word regions (SWRs). (f) Merged image (WRs). (g) Splitting overlapping CC.

Consequently, the proposed method for dividing the text into words or word structures was adopted to avoid the problems of using the techniques for dividing the text into lines and then into words, in proportion to the database through the following stages:

a) Read all original pictures of each writer and find their Negative. "Figure 2" represents the negative original image.

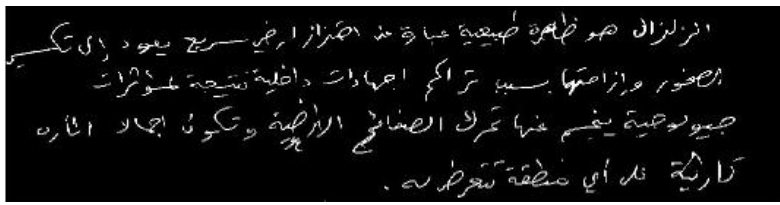

Figure 2: Negative orginal image

b) Determining all the connected components (CCs connected-components) from the binary image, according to the algorithm presented by Haralick et al [21].

c) The height of the connected components is Calculated experimentally to adopt the most frequent height to ensure that no words or word structures are captured from several lines.

d) Applying the (LoG: Laplacian of Gaussian) filter to the matrix of connected components, which resulting from phase (b). The filter parameters were adopted after several experiments according to the following (size $=$ $[7,7]$, sigma $=0.1 * \mathrm{~h}$ ), the "Figure $3 "$ represents the image after filtering.

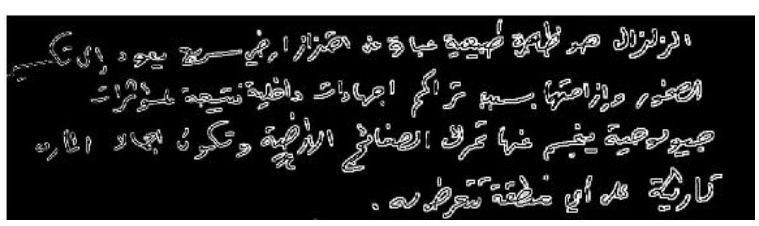

Figure 3: Image after filtering e) Filling the filtered image, by using an algorithm based on morphological reconstruction, as shown in "Figure 4" [22].

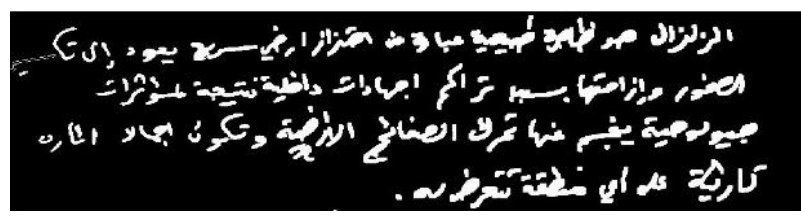

Figure 4: Filling image regions and holes after filtering

f) Determining all the connected components (CCs: connected-components) from the output image of phase (e).

g) Determining the connected components, which resulting from phase (f) on the original image as shown in "Figure $5 "$

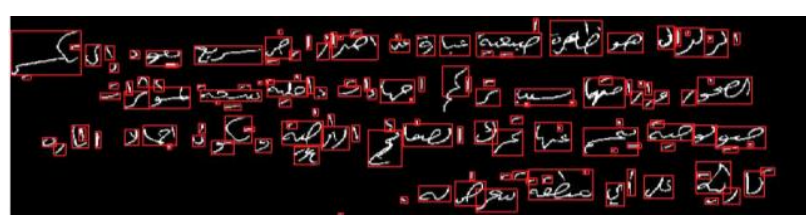

Figure 5: Determining connected components on image shown in Fig (2)

h) Deleting the connected components that are within larger connected components and make the interconnected components a single component that includes them as shown in "Figure 6"

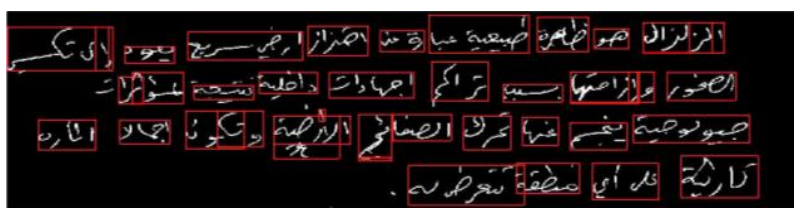

Figure 6: Delete connected components that are among other components

2) Collecting all the words or word structures Randomly, which resulting from the previous stage in one line and repeat this step to get several different lines, according to the following stages:

a) Reading images of words or word structures resulting from the first stage of each writer and "Figure 7" shows us some pictures of words and word structures for one of the writers, to note that the dividing text into words has picked up words, parts of words, and sometimes word structures. This is due to the nature of Writing in Arabic language, which some of its words may be composed of separate parts, and to the writing style of the writer.

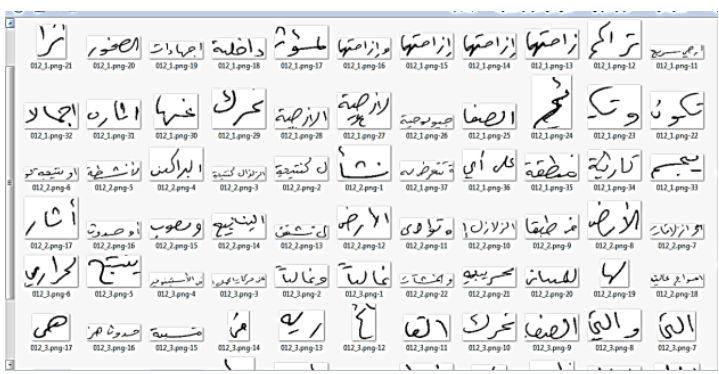

Figure 7: sample of word pictures and word structures for a writer 
b) Determining the size of the line images by taking the sum of the width of each image resulting from the previous stage (a) and separating them by three pixels and adopting the largest height of the images resulting fromthe previous stage (a) is the height of the line images.

c) Generating a number of line images by randomly combining the images resulting from stage (a) in order to obtain hundreds of different line images from each other, as shown in "Figure 8".

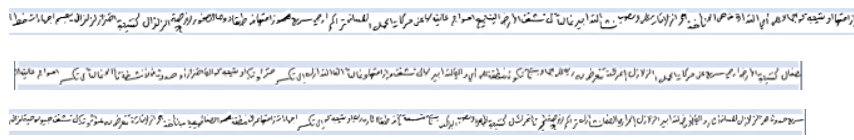

Figure 8: Sample of some generated text line images

3) Forming an image of a five-line text generated from stage (c) in the second stage, as shown in "Figure 9", in order to ensure the presence of a number of words not less than 15 words, based on the study presented by $\mathrm{Wu}$ et al [23].

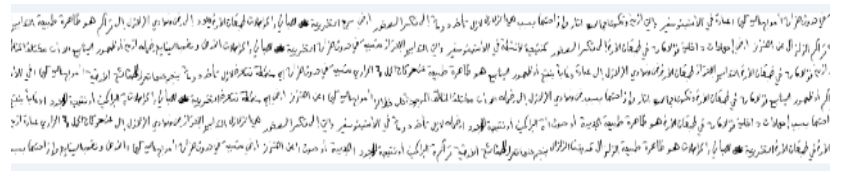

Figure 9: sample Image of a 5-line generated text

4) Croping each image generated from the third stage into a number of images with a size of $400 * \mathrm{R}$, where (R) represents the height of the image generated from the third stage, as shown in "Figure 10", and then the size of all images was standardized when entered into the deep learning network .

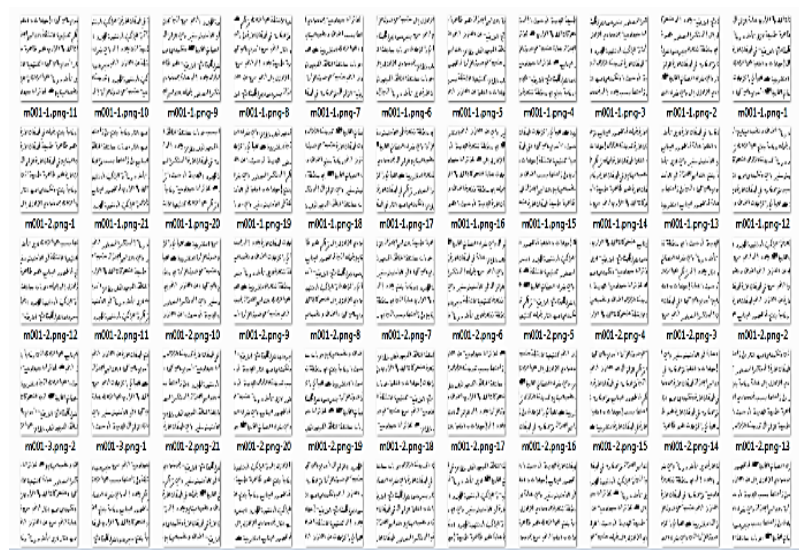

Figure 10: sample of the text images after croping

Hundreds of pictures of handwriting texts for each writer are got from this stage; the first 200 handwriting images resulting from Data Augmentation stage for each writer were taken to obtain a database of 40400 handwriting scripts for 202 writers and for each writer 200 texts.

\subsection{Convolutional neural network (CNN)}

In Deep Learning research, CNNs are specifically applied for Computer Vision applications that involves Image
Classification and Object Recognition.Convolutional neural network's architecture has several famous designs, including:LeNet, AlexNet, GoogLeNet, VGGNet.

A Convolutional Neural Network (CNN) has three types of layers as follows: Convolutional Layer (CONV), Pooling Layer (POOL), and Fully-Connected Layer (FC).

\subsubsection{Convolutional Layer (CONV)}

This is the most important layer in any CNN architecture because this is the layer where CNN applies filters to learn features from the input image. There are three hyperparameters, that are required to tune the CONV layer. They are depth, stride and zero-padding.

Thus, in a CONV layer, the input volume is represented as [ $\left.\mathrm{W}_{1} \mathrm{x} \quad \mathrm{H}_{1} \mathrm{x} \mathrm{D}_{1}\right]$ corresponding to the spatial dimensions of the input image. Four hyperparameters are represented as $[\mathrm{K}, \mathrm{F}$, $\mathrm{S}, \mathrm{P}]$ corresponding to the number of filters, the size of the filter, the stride and the amount of zero padding. The output volume is represented as [W2 $\times \mathrm{H} 2 \times \mathrm{D} 2]$ corresponding to:
$\mathrm{W}_{2}=\left(\mathrm{W}_{1}-\mathrm{F}+2 \mathrm{P}\right) / \mathrm{S}+1$
$\mathrm{H}_{2}=\left(\mathrm{H}_{1}-\mathrm{F}+2 \mathrm{P}\right) / \mathrm{S}+1$
$\mathrm{D}_{2}=\mathrm{K}$

\subsubsection{Pooling Layer (POOL)}

This layer is used as an intermediate layer in the network where it downsamples or compresses the incoming volume along the spatial dimensions. Thus, it downsamples the previous layer's feature maps obtained from different filters to reduce computations in the network.

Thus, in a POOL layer, the input volume is represented as $\left[\begin{array}{lll}W_{2} \times H_{2} \times & D_{2}\end{array}\right]$ corresponding to the spatial dimensions of the input volume, two hyperparameters are represented as $\left[\mathrm{F}_{1}, \mathrm{~S}_{1}\right]$ corresponding to the receptive field or size of the filter and the stride, and the output volume is represented as $\left[\mathrm{W}_{3} \mathrm{x} \mathrm{H}_{3} \mathrm{x}\right.$ $\mathrm{D}_{3}$ ] corresponding to:
$\mathrm{W}_{3}=\left(\mathrm{W}_{2}-\mathrm{F}\right) / \mathrm{S}+1$
$\mathrm{H}_{3}=\left(\mathrm{H}_{2}-\mathrm{F}\right) / \mathrm{S}+1$
$\mathrm{D}_{3}=\mathrm{D} 2$

\subsubsection{Fully-Connected Layer $(F C)$}

As in ANNs, the FC layer in a CNN has neurons that are fully connected to the neurons in the previous layer. This FC layer is often kept as the final layer of a CNN with "SOFTMAX" as its activation function for multi-class classification problems. The FC layer is responsible to predict the final class or label of the input image. Thus, it has an output dimension of $[1 \mathrm{x} 1 \mathrm{xN}]$ where $\mathrm{N}$ denotes the number of classes or labels considered for classification [24].

The network architecture in this research consists of 4 convolutional layers, 4 pooling layers, and 2 Fully Connected Layer (FC). Each convolutional layer is followed by a Batch Normalization Layer (BN) and Relu layer. This network also includes a Softmax layer, which is used for Classification problems.

The description of this architecture convolutional network layers is as follows and illustrated according to "Figure 11":

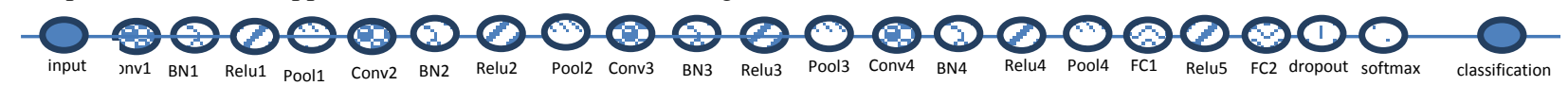

Figure 11: The structure of the convolutional network 
1. The input layer receives training samples, which is binary images and size $[400,400]$.

2. Convolutional Layer (CONV1): In this layer, 96 filters of size $\mathrm{F}=[5,5]$ were used, which values are assumed by independently sampling from a normal distribution with zero mean and standard deviation 0.01 during training initially, and stride $(S=2)$ without applying zero padding.

3. Batch Normalization Layer: It normalizes input by mini batch, speeds up convolutional neural network training and reduces the sensitivity of network configuration. Therefore, batch normalization layers are used between convolutional and nonlinear layers, such as ReLU layers.

4. Relu Layer: This layer applies a threshold to all its input elements coming from the previous layer according to the function shown below:

$f(x)= \begin{cases}x, & x \geq 0 \\ 0, & x \leq 0\end{cases}$

5. The pooling layer: (Pooling Layer (Pool1)) In this layer, Max Pooling was used, meaning the maximum grouping and the size of the area from which the maximum value will be chosen is of size $\mathrm{F}=[2,2]$, and a stride $(\mathrm{S}=2)$.

6. Convolutional Layer (CONV2): In this layer, 128 filters of size $\mathrm{F}=[3,3]$ were used, which values are assumed by independently sampling from a normal distribution with zero mean and standard deviation 0.01 during training initially during training initially, and stride $(S=2)$ without applying zero padding.

7. Batch Normalization Layer.

8. Relu Layer.

9. The pooling layer: (Pooling Layer (Pool2)): The size of the area from which the maximum value will be chosen is of size $F=[2,2]$, and a stride $(S=2)$.

10. Convolutional Layer (CONV3): In this layer, 256 filters of size $\mathrm{F}=[3,3]$ were used, which values are assumed by independently sampling from a normal distribution with zero mean and standard deviation 0.01 during training initially during training initially, and stride $(S=1)$ without applying zero padding.

11. Batch Normalization Layer.

12. Relu Layer.

13. The pooling layer: (Pooling Layer (Pool3)): The size of the area from which the maximum value will be chosen is of size $F=[2,2]$, and a stride $(S=2)$.

14. Convolutional Layer (CONV4): In this layer, 300 filters of size $\mathrm{F}=[1,1]$ were used, which values are assumed by independently sampling from a normal distribution with zero mean and standard deviation 0.01 during training initially during training initially, and stride $(S=1)$ without applying zero padding.

15. Batch Normalization Layer.

16. Relu Layer
17. The pooling layer: (Pooling Layer (Pool4)): The size of the area from which the maximum value will be chosen is of size $F=[2,2]$, and a stride $(S=2)$.

18. Fully Connected Layer (FC1): The FC layer contains neurons fully connected to the neurons in the previous layer, the output volume of this layer has been determined (450)

19. Relu Layer.

20. Dropout Layer: This layer effectively changes the network structure between iterations and helps prevent the network from over-fitting by setting the input elements randomly to zero according to the probability that was determined when building the network and the most appropriate probability value for this network is $(0.5)$.

21. Fully Connected Layer (FC2): The output size has been specified for this layer (202), which represents the number of classes.

22. Softmax Layer Activation Function: It is used in classification problems for several classes. In classification problems. A softmax layer and a classification layer must follow the last fully connected layer, where the activation function of the output unit is the softmax function.

23. Classification Layer.

\section{EXPERIMENTS AND RESULTS}

The experiments were conducted on Matlab version R2019a. The database includes 202 writers and each writer has 3 texts to be increased by using the Data Augmentation techniques proposed in this research, so that each writer has 200 texts and the dataset becomes 40400 handwriting images. The dataset was divided into 3 groups $90 \%$ as a training set, chose $5 \%$ of them as a validation set, and $10 \%$ as a test set. Training the network on the training samples, by assuming the weights values initially through sampling from a normal distribution with zero mean and standard deviation 0.01 with Max Epoch value equal to 5 . The two Schematic diagram shown in "Figure 12" and "Figure 13" the first express the values of training accuracy and the other the loss values during the training stage.

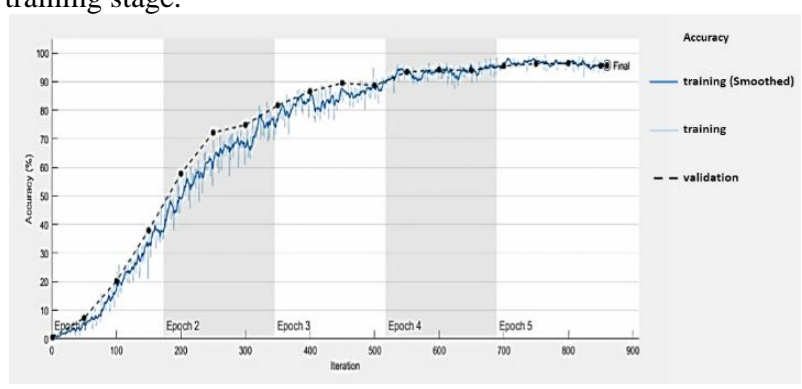

Figure 12: The changes in the accuracy of the training and validation samples during the training stage at Max Epoch $=5$

Let us note in the beginning of the training that the accuracy values are small to increase at the end of the training stage. The accuracy values of the validation samples are greater than the accuracy of the training samples in the beginning to become convergent at the end of the training and this study obtain a value $95 \%$ for the validation samples and $95.8196 \%$ for the training samples. 


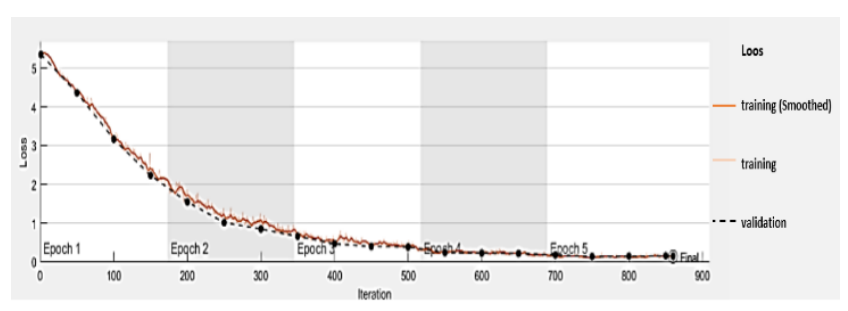

Figure 13: The changes in the loss of the training and validation samples during the training stage at Max Epoch $=5$

Let us notice at the beginning of the training that the loss values are large to decrease at the end of the training stage. The loss values of the validation samples are less than the loss values of the training samples to become convergent at the end of the training and this study obtain a value 0.1339 for the validation samples and 0.1375 for the training samples.

To know the effectiveness of the proposed method, the accuracy was measured using relationship (4.1) to obtain $96.5099 \%$ classification accuracy.

Precision $=\frac{\text { True Positives }}{\text { True Positives+ FalsePositives }} * \mathbf{1 0 0}$

The network was retrained on the trained weights from the previous stage, and the Max Epoch value was set to 3 to see the network training path according to the two diagrams shown in "Figure 14" and "Figure 15" to obtain classification accuracy of $98.2426 \%$.

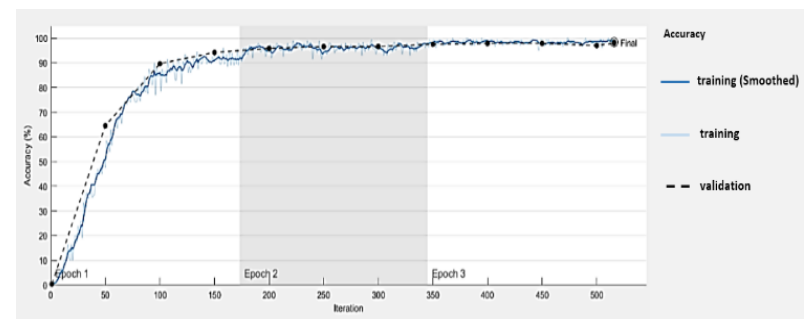

Figure 14: The changes in the accuracy of the training and validation samples during the training stage at Max Epoch $=3$

Note that the previously trained weights gave an improvement in the accuracy values, which played a role in detecting features from the handwriting text images.

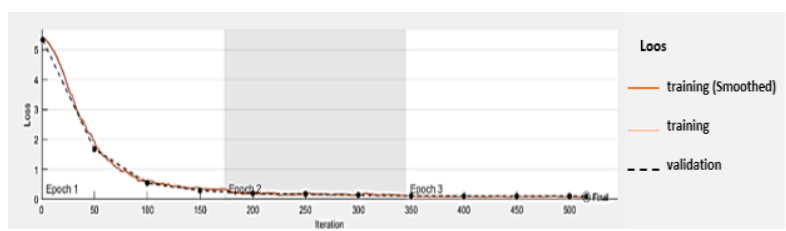

Figure 15: The changes in the loss of the training and validation samples during the training stage at Max Epoch $=3$

Note that the previously trained weights gave an improvement in the loss values, which played a role in detecting the features from the handwriting text images.

\section{CONCLUSION AND FUTURE WORK}

Writer identification is a relatively new biometric modality that has received significant research attention in the recent years. Handwriting biometrics can be used in the forensic applications to identify individuals based on their writing characteristics by comparing unlabeled handwritten texts with labeled handwritten samples.

This research provided CNN model to know more distinctive information from handwriting pictures, that is more powerful to represent characteristics of different writing styles to improve writer identification performance.

The proposed method has several advantages. First, the data augmentation technique is programmed to avoid restricting a small amount of handwriting samples. Second, the characteristics of each writer are obtained and recognized based on the CNN model from the handwritten images instead of using special methods to extract the features, and then apply one of the methods to identify the writer. The proposed method gets the best performance on the data set, which shows the effectiveness of the proposed method for writer identification.

In the future, experiments will be focused interest on applying this study to a new and more diverse database of texts that contain structures in Arabic and English and numbers, in order to determine the identification accuracy with these cases in the text.

\section{REFERENCES}

[1] SREERAJ, M., IDICULA, S., 2011- A Survey on Writer Identification Schemes. International Journal of Computer Applications, (26).

[2] SARANYA, K., VIJAYA, M. S., 2013- Text Dependent Writer Identification using Support Vector Machine. International Journal of Computer Applications, (65).

[3] TOMAI, C., ZHANG, B., SRIHARI, S.2004Discriminatory Power of Handwritten Words for Writer Recognition. Proc. 17th Int'1 Conf. Pattern Recognition, pp. 638-641.

[4] ZHU, G., YU, X., LI, Y., DOERMANN, D. 2009 Language identification for handwritten document images using a shape codebook. Pattern Recogn.(42), 3184-3191

[5] Li,B., Sun, Z., Tan,T.N., 2009- Hierarchical Shape Primitive Features for Online Text-independent Writer Identification. Proc. of 2th ICB, pages 201-210.

[6] DJEDDI, C., SIDDIQI, I., MESLATI, L., ENNAJI, A. 2013- Text-independent writer recognition using multiscript handwritten texts. journal homepage, 1196-1202.

[7] ALAEI,A., ROY, P.P., 2014- A New Method for Writer Identification based on Histogram Symbo lic Representation. 14th International Conference on Frontiers in Handwriting Recognition, 2167-6445.

[8] THENDRAL, T., VIJAYA, M. S., KARPAGAVALLI, S., 2015- Prediction of Writer Using Tamil Handwritten Document Image Based on Pooled Features. International Journal of Computer, Electrical, Automation, Control and Information Engineering, (9).

[9] AL-ZOUBEIDY ,L. M. AL-NAJAR ,H. F., 2005Arabic writer identification for handwriting images, International Arab Conference on Information Technology, pp. 111-117.

[10] Gazzah, S., Amara, N.E, 2007- Arabic Handwriting Texture Analysis for Writer Identification using the DWT-lifting Scheme. In 9th ICDAR,(2), 1133-1137. 
[11] CHEN, J., LOPRESTI, D., KAVALLIERATOU,E., 2010- The Impact of Ruling Lines on Writer Identification. In: 12th International Conference on Frontiers in Handwriting Recognition, pp. 439 - 444.

[12] SLIMANE ,F., MARGNER, V., 2014- A New TextIndependent GMM Writer Identification System Applied to Arabic Handwriting. 14th International Conference on Frontiers in Handwriting Recogniti.

[13] MESLATI, L., ENNAJI, A., DJEDD, CH., 2012- Writer Recognition on Arabic Handwritten Documents. Springer-verlag berlin, Heidelberg, (7340),pp.493-501.

[14] DJEDDI, CH., MESLATI, L., SIDDIQI,I., ENNAJI,A., ABED,H., GATTAL,A., 2014- Evaluation of Texture Features for Offline Arabic Writer Identification. 11th IAPR International Workshop on Document Analysis Systems.

[15] BENNOUR A.,2018 - Clonal Selection Classification Algorithm Applied to Arabic Writer Identification .Proceedings of ACM ICIST conference, Istanbul, Turkey, March 2018 (ICIST '18), 5 pages.

[16] DENGEL,A., LIWICKI,M., RASHID,S., AFZAL,M., NAZ,S., AHMAD,R. 2018 - A Deep Learning based Arabic Script Recognition System: Benchmark on KHAT. The International Arab Journal of Information Technology, Vol. 17, No. 3, May 2018.
[17] REHMAN,A., NAZ,S., RAZZAK,M., 2019 - Automatic Visual Features for Writer Identification: A Deep Learning Approach. IEEE Volume 7, 2019.

[18] www.kaggle.com/c/awic2012/leaderboard

[19] TANG,Y., WU,X., BU,W., Member IEEE , 2014 Offline Text-Independent Writer Identification Based on Scale Invariant Feature Transform. IEEE Transactions On Information Forensics And Security, Vol. 9, No. 3, March 2014.

[20] Zheng,Y., Li,H., Doermann,D, 2002 - The Segmentation and Identification of Handwriting in Noisy Document Images Transform. Springer 2423, pp. 95-105, 2002.

[21] Haralick, Robert M., and Linda G. Shapiro, Computer and Robot Vision, Volume I, Addison-Wesley, 1992, pp. 28-48.

[22] Soille, P., Morphological Image Analysis: Principles and Applications, Springer-Verlag, 1999, pp. 173-174.

[23] TANG,Y., WU,X., 2016 - Text-independent Writer Identification via CNN Features and Joint Bayesian. IEEE, 2016 15th International Conference On Frontiers In Handwriting Recognition.

[24] Gogul.,Kumar,S., 2017- Flower Species Recognition System using Convolution Neural Networks and Transfer Learning. 2017 IEEE, 2017 4th International Conference on Signal Processing, Communications and Networking. 